\title{
Study on the cost attributable to central venous catheter-related bloodstream infection and its influencing factors in a tertiary hospital in China
}

\author{
Yuanyi Cai ${ }^{1}$, Min Zhu', Wei Sun ${ }^{2}$, Xiaohong Cao ${ }^{1}$ and Huazhang Wu ${ }^{1 *}$
}

\begin{abstract}
Background: Central venous catheters (CVC) have been widely used for patients with severe conditions. However, they increase the risk of catheter-related bloodstream infection (CRBSI), which is associated with high economic burden. Until now, no study has focused on the cost attributable to CRBSI in China, and data on its economic burden are unavailable. The aim of this study was to assess the cost attributable to CRBSI and its influencing factors.

Methods: A retrospective matched case-control study and multivariate analysis were conducted in a tertiary hospital, with 94 patients (age $\geq 18$ years old) from January 2011 to November 2015. Patients with CRBSI were matched to those without CRBSI by age, principal diagnosis, and history of surgery. The difference in cost between the case group and control group during the hospitalization was calculated as the cost attributable to CRBSI, which included the total cost and five specific cost categories: drug, diagnostic imaging, laboratory testing, health care technical services, and medical material. The relation between the total cost attributable to CRBSI and its influencing factors such as demographic characteristics, diagnosis and treatment, and pathogenic microorganism, was analysed with a general linear model (GLM).
\end{abstract}

Results: The total cost attributable to CRBSI was $\$ 3528.6$, and the costs of specific categories including drugs, diagnostic imaging, laboratory testing, health care technical services, and medical material, were $\$ 2556.4, \$ 112.1$, \$321.7, \$268.7, \$276.5, respectively. GLM analysis indicated that the total cost was associated with the intensive care unit (ICU), pathogenic microorganism, age, and catheter number, according to the sequence of standardized estimate ( $\beta$ ). ICU contributed the most to the model R-square.

Conclusion: Central venous catheter-related bloodstream infection represents a great economic burden for patients. More attentions should be paid to further prevent and control this infection in China.

Keywords: Catheter-related bloodstream infection, Cost, Influencing factor

\section{Background}

Central venous catheters $(\mathrm{CVC})$ have been widely used in the treatment of patients with severe diseases. However, the utilization of CVC may cause catheter-related bloodstream infection (CRBSI), which is a great economic burden on the patients. In recent years, many studies have

\footnotetext{
* Correspondence: hzwu@cmu.edu.cn

${ }^{1}$ Department of Health Service Management, School of Humanities and Social Sciences, China Medical University, No.77 Puhe Road, Shenyang North New Area, Shenyang, Liaoning Province 110122, People's Republic of China Full list of author information is available at the end of the article
}

been carried out worldwide to focus on the cost of CRBSI. According to our knowledge, four designs including a retrospective matched case-control study [1-3], prospective research [4-8], multivariate analysis [9], and a case report [10], have been used to evaluate the cost attributable to CRBSI. The cost of CRBSI has been estimated to be between $\$ 11,971$ and $\$ 69,332$ in America $[1,4,5,9,10]$. Studies performed in European countries have reported the cost to be in the ranges of $€ 13,585$ to $€ 29,909[2,6]$. The value calculated in developing countries has ranged

C The Author(s). 2018 Open Access This article is distributed under the terms of the Creative Commons Attribution 4.0 International License (http://creativecommons.org/licenses/by/4.0/), which permits unrestricted use, distribution, and reproduction in any medium, provided you give appropriate credit to the original author(s) and the source, provide a link to the Creative Commons license, and indicate if changes were made. The Creative Commons Public Domain Dedication waiver (http://creativecommons.org/publicdomain/zero/1.0/) applies to the data made available in this article, unless otherwise stated. 
between $\$ 4888$ and $\$ 11,591[7,8]$. A recent Japanese study reported an excess cost of $\$ 57,090$ for CRBSI [3].

In China, CRBSI is currently one of the four nosocomial infections monitored by the Chinese government [11], and it has aroused increasing public attention. A prospective investigation of CRBSI was conducted across 12 provinces and showed that the incidence rate was between 0.69 and 14.22 per 1000 central catheter days (133 out of 4256 patients infected by CRBSI in 55 intensive care units (ICU) from 41 hospitals monitored from October 2013 to March 2014) [12]. However, there is still no report about the cost attributable to CRBSI in China. The factors influencing this economic burden are also unknown. The Chinese government is currently trying to solve the problem of the high costs of obtaining medical services with new medical reform. Distinguishing between the cost of primary disease and that of CRBSI can help the government to determine the origin of the economic burden and further evaluate the burden from CRBSI itself, which would be useful to establish targeted control strategies. Furthermore, an analysis of factors that influence the cost attributable to CRBSI will also provide evidence to hospitals for improving medical quality and to patients for exerting better self-care during hospitalization.

The purpose of the present study was to assess the cost attributable to CRBSI and explore its influencing factors for the government, hospitals and patients. Because the incidence of CRBSI is low in China [12], and the information in Chinese hospital medical records is not as sufficient as that in developed countries, methods such as prospective research, multivariate analysis, and case reports were not feasible. Thus, this study was a retrospective matched case-control study. The cost attributable to CRBSI and its influencing factors available in the hospital information system (HIS) and medical records, including demographic characteristics, diagnosis and treatment, and pathogenic microorganisms, were analysed. We believe our findings can help the government, hospitals and patients to prevent and control this infection.

\section{Methods}

\section{Definition}

CRBSI is described as the presence of bacteraemia or fungemia in patients with a catheter or within $48 \mathrm{~h}$ after the catheter removal without the presence of any other source of infection. The clinical signs of patients with CRBSI are fever $\left(>38{ }^{\circ} \mathrm{C}\right)$, chills, and hypotension. Laboratory microbiology examinations have shown a positive result for the peripheral blood sample culture or the catheter tip culture of the same microorganism [13].

\section{Study population}

The study was conducted in a tertiary hospital affiliated with China Medical University. It is a public 3346-bed hospital. All patients (age $\geq 18$ years old) with central venous catheter-related bloodstream infection in every department from January 2011 to November 2015 were included, and there were a total of 94 patients for the case group. Meanwhile, a matched (1:1) case-control study design was utilized. The controls were defined as patients with CVC insertion but without CRBSI. A total of three key variables were used in the matching procedure, including age ( $\pm 10 \mathrm{yr}$.), principal diagnosis, and history of surgery $[8,14]$.

\section{Assessment of cost attributable to CRBSI}

The cost data were derived from the patients' true hospital expenditure and were provided by the financial department of the hospital. The duration from the time of admission of the patient to the day of discharge was selected. The costs of patients with CRBSI were analysed and compared with those of the patients without CRBSI. The difference in cost between the two groups during the hospitalization was calculated as the cost attributable to CRBSI. This difference included the total cost and five specific cost categories: 1) drug, 2) diagnostic imaging, 3) laboratory testing, 4) health care technical service, and 5) medical material. The analysis of the drug included only western medicine. Diagnostic imaging included the diagnosis of Doppler ultrasonography, electrocardiogram, computed tomography (CT), and other small medical devices. Laboratory testing mainly included a routine blood test, and a bacterial culture. Health care technical service included surgery, physical examination, nursing, medical disposition, and group consultation. Medical material was defined as the medical consumables and equipment used in the hospital such as catheters and sutures. The currency exchange rate used was US\$1 to $¥ 6.8977$.

\section{Measurements of demographic characteristics, diagnosis and treatment, and pathogenic microorganism}

Demographic characteristics included age, sex, occupation, and immunosuppressive drug history. Occupation was categorized as worker, farmer, office clerk, retiree, and others according to the information provided by the patients on the front page of the medical record.

Diagnosis and treatment was assessed based on 5 items: 1) ICU, 2) principal diagnosis, 3) other diagnoses, 4) operation level, and 5) catheter number. If the patients were admitted to the ICU during the hospital stay, this indicated that they had a severe condition and that their treatment would cost much more than those of other patients. The principal diagnosis was categorized as cancer, inflammation, and others. Patients with cancer and severe inflammation made an impact on the treatment of CRBSI. Other diagnoses were marked "yes" if diagnoses such as hypertension and diabetes were found in the patients' medical records. The operation level was categorized as not performing an operation or performing an operation 
and ranged from level one to level four based on the complexity of the surgery [15], with level four representing the most difficult operation. The catheter number was assessed through the total number of $\mathrm{CVC}$, tracheal cannula, urinary catheter, drainage tube, and stomach tube. Patients with few (less than or equal to four) catheters were combined with patients with five catheters into " $\leq 5$ group".

Pathogenic microorganisms were categorized as gram-positive bacteria $(\mathrm{G}+)$, gram-negative bacteria (G-), and fungi to explore their impact on the cost.

\section{Statistical analysis}

The difference in age between the two groups after the matching procedure was evaluated by Student's t-test. The difference in cost for the patients in the case and control group and the different influencing factors in the univariate analysis were tested by the Wilcoxon rank sum test. General linear model analysis was used to clarify the influencing factors for the total cost attributable to CRBSI. Variables significant at the 0.25 level in the univariate analysis were entered in the model. Items with $P>0.15$ were eliminated one at a time in the sequence of $P$ value. When a variable was eliminated, it remained in the model as a confounder if any remaining parameter estimate changed over $20 \%$. In the study, no confounder was found during elimination. SAS for Windows (V8.2) was used for the statistical analyses.

\section{Results}

\section{Fundamental results of matching}

According to the matching criteria, 81 patients were successfully matched with control patients (the information of the other 13 patients could not be retrieved through the HIS). The average age of the case group was $62.2 \pm 11.2$ years old, and that of the control group was $61.9 \pm 11.3$ years old $(P=0.867)$. Twenty-five types of principal diagnosis were involved. Pancreatic tumor (14 pairs), rectal cancer (10 pairs), cholangiocarcinoma (9 pairs), gastric cancer (8 pairs), and liver cancer (5 pairs) were the main primary diseases, and made up $56.8 \%$ of all pairs. Considering the history of surgery, twenty-one types of operations associated with the primary diseases were performed.

\section{Evaluation of the cost attributable to CRBSI}

The median cost in the CRBSI group was $\$ 12,515.2$, which was significantly higher $(P<0.001)$ than that in the control group. The total median cost attributable to CRBSI was $\$ 3528.6$. Furthermore, the differences between the drug costs, diagnostic imaging costs, laboratory testing costs, technical fees, and medical material costs of the two groups were \$2556.4, \$112.1, \$321.7, $\$ 268.7$, and $\$ 276.5$, respectively (Table 1 ).

\section{Influencing factors of the cost attributable to CRBSI}

The characteristics and distributions of the total cost attributable to CRBSI are shown in Table 2. There were significant differences in the ICU factors $(P<0.001)$ and catheter numbers $(P=0.002)$ of the patients. In addition, there was a mild correlation between the total cost and other diagnoses $(P=0.053)$.

The results of the general linear model analysis for clarifying the factors influencing the economic burden attributable to CRBSI are shown in Tables 3 and 4. The additional cost was significantly related to ICU, pathogenic microorganism, age, and catheter number according to the sequence of the standardized estimate $(\beta)$. The contribution to the model R-square attributable to the four factors $(\Delta \mathrm{R}$-square) was $0.1488,0.0326,0.0339$, and 0.1078 , respectively.

\section{Discussion}

This study is the first to report the cost attributable to CRBSI and its influencing factors in China. Our results revealed that the median cost attributable to CRBSI was $\$ 3528.6$ and was only lower than those of the five most costly diseases, which were myocardial infarction-coronary artery bypass grafting (\$9700.9), acute myocardial infarction (\$4901.9), gastric cancer (\$4101.2), lung cancer (\$3785.1), and esophageal cancer (\$3601.9) according to the list of 30 diseases whose economic burden was evaluated by the $\mathrm{Na}$ tional Health Commission [16]; however, the cost attributable to CRBSI was more than that of the other diseases.

In addition, the cost attributable to CRBSI was 2.3 times that of the per capita net income (\$1561.7) of the farmers

Table 1 Medical costs attributable to CRBSI (\$)

\begin{tabular}{lllll}
\hline & Median cost in the CRBSI group & Median cost in the control group & Medical cost attributable to CRBSI & $P$ \\
\hline Total & $12,515.2$ & 8450.5 & 3528.6 & $<0.001$ \\
Drug & 6576.0 & 4056.9 & 2556.4 & $<.001$ \\
Diagnostic imaging & 401.3 & 328.8 & 112.1 & $<0.001$ \\
Laboratory testing & 906.1 & 536.0 & 321.7 & $<0.001$ \\
Technical fees & 1325.7 & 987.9 & 268.7 & $<0.001$ \\
Medical material & 1697.3 & 1369.4 & 276.5 & 0.002 \\
\hline
\end{tabular}


Table 2 The characteristics and distributions of the total cost attributable to CRBSI

\begin{tabular}{|c|c|c|c|c|}
\hline Variables & & $n$ & Median(\$) & $P$ \\
\hline \multirow[t]{2}{*}{ Age(yr.) } & $\leq 59$ & 33 & 2977.9 & 0.152 \\
\hline & $>60$ & 48 & 4424.3 & \\
\hline \multirow[t]{2}{*}{ Sex } & Male & 50 & 3070.5 & 0.290 \\
\hline & Female & 31 & 4042.8 & \\
\hline \multirow[t]{5}{*}{ Occupation } & Worker & 7 & 1902.5 & 0.702 \\
\hline & Farmer & 17 & 2977.9 & \\
\hline & Office clerk & 9 & 3528.6 & \\
\hline & Retiree & 30 & 4436.9 & \\
\hline & Others & 18 & 3495.1 & \\
\hline \multirow[t]{2}{*}{ Immunosuppressive drug history } & No & 69 & 3458.8 & 0.481 \\
\hline & Yes & 12 & 5316.0 & \\
\hline \multirow[t]{2}{*}{ ICU } & No & 65 & 2420.7 & $<0.001$ \\
\hline & Yes & 16 & 9154.3 & \\
\hline \multirow[t]{3}{*}{ Principal diagnosis } & Cancer & 59 & 3087.0 & 0.218 \\
\hline & Inflammation & 6 & 8760.0 & \\
\hline & Others & 16 & 4368.9 & \\
\hline \multirow[t]{2}{*}{ Other diagnoses } & No & 28 & 1930.6 & 0.053 \\
\hline & Yes & 53 & 4042.8 & \\
\hline \multirow[t]{3}{*}{ Operation level } & $\leq 2$ & 24 & 2920.2 & 0.228 \\
\hline & 3 & 24 & 6002.2 & \\
\hline & 4 & 33 & 3087.0 & \\
\hline \multirow[t]{2}{*}{ Catheter number } & $\leq 5$ & 64 & 2549.0 & 0.002 \\
\hline & 6 & 17 & 7587.4 & \\
\hline \multirow[t]{3}{*}{ Pathogenic microorganism } & G- & 25 & 2335.6 & 0.137 \\
\hline & G+ & 42 & 3601.9 & \\
\hline & Fungus & 14 & 6340.1 & \\
\hline
\end{tabular}

and made up $78.0 \%$ of the disposable income of the urban residents (\$4522.5) in China [17]. Therefore, the occurrence of CRBSI further increased the economic burden for the patients, especially for those from rural areas or with limited insurance coverage and reimbursement.

The cost attributable to CRBSI in this study was significantly lower than the level of developed countries and slightly lower than that of developing countries. Three reasons contribute to the differences. First, unlike the studies in other countries, our study showed that most patients in this sample hospital were from general wards $(80.2 \%)$ rather than from ICUs, which decreased the cost to some extent. Second, there are significant differences in economic development and medical price across the countries, which may impact the cost. Third, the unsound health care pricing system in China is another reason. The value of the medical staff is greatly underestimated. In the specific cost categories, our study

Table 3 The general linear model analysis for clarifying the major factors influencing the economic burden attributable to CRBSI

\begin{tabular}{|c|c|c|c|}
\hline Variables & Parameter estimate (B) & Standardized estimate $(\beta)$ & Model R-Square \\
\hline Intercept & 3997.14 & & \\
\hline ICU (Yes vs. No) & $4962.37^{* * *}$ & 0.40 & \\
\hline Pathogenic microorganism (G+ vs. fungus) & $-3315.04^{* *}$ & -0.31 & \\
\hline Pathogenic microorganism (G- vs. fungus) & $-2246.35^{*}$ & -0.23 & \\
\hline Age (yr.) (> 60 vs. $\leq 59)$ & $2154.24^{* *}$ & 0.21 & \\
\hline Catheter number ( 6 vs. $\leq 5)$ & $2196.67^{*}$ & 0.18 & $0.3231^{* * *}$ \\
\hline
\end{tabular}

${ }^{\bar{*} P} P<0.15 .{ }^{* *} P<0.05 .{ }^{* * *} P<0.01$ 
Table 4 The contributions of factors of the model R-square

\begin{tabular}{|c|c|c|c|c|}
\hline & Demographic characteristics & Diagnosis and treatment & & Pathogenic microorganism \\
\hline \multicolumn{5}{|c|}{ Statistic } \\
\hline & Age & & & \\
\hline & & Catheter number & & \\
\hline & & & ICU & \\
\hline & & & & Pathogenic microorganism \\
\hline $\mathrm{F}$ & 3.81 & $7.6^{* *}$ & $11.92^{* *}$ & $8.64^{* *}$ \\
\hline$R^{2}$ & 0.0339 & 0.1417 & 0.2905 & 0.3231 \\
\hline$\Delta \mathrm{R}^{2}$ & 0.0339 & 0.1078 & 0.1488 & 0.0326 \\
\hline
\end{tabular}

${ }^{* *} P<0.01$

revealed that the median drug cost was the main component. However, the median health care technical fee, which reflected the value of the medical staff, was underestimated and only made up $7.6 \%$ of the total additional cost. The unsound pricing system is one of the structural problems in the health care system in China and needs to be changed with the new medical reform.

The influencing factors of age, catheter number, ICU, and pathogenic microorganisms were found to be crucial. Moreover, the ICU was found to have the strongest association with the cost caused by CRBSI among all four factors, which made up the major contribution (0.1488) to the model R-Square. In the ICU, patients with severe situations need special care, sophisticated medical monitoring devices, and better drugs, all of which lead to higher expenditure for the treatment of CRBSI. Furthermore, the price elasticity of demand is lower in the ICU because the patients and relatives are faced with extremely limited options concerning life and health [18]. The number of catheters was another important factor influencing the cost attributable to CRBSI. Other kinds of catheters were used such as tracheal cannula, urinary catheter, drainage tube, and stomach tube, and other types of infections might occur, which makes the recovery of CRBSI much more difficult [19].

Both age and pathogenic microorganisms, contributed less to the model R-Square. Unlike those of young patients, the organ function and immune system of old patients decline, which makes treatment more difficult [20]. China is becoming an ageing society. It is clear that the health care expenditures of the whole nation will be significantly increased [21] if CRBSI cannot be prevented in elderly patients. Comparing the pathogenic microorganisms, fungal infections had a much higher impact on cost than G+ and G- bacterial infections. The result was consistent with the study of Itaru Nakamura, who reported that Candida CVC-CRBSI incurred the highest cost. This was likely due to the broader consequences of bloodstream fungal infection such as severe sepsis and multiple organ failure [3].
There are two limitations in the study. First, the lower incidence of CRBSI in China resulted in a small sample size in our study, which had an impact on the cost results and influencing factor analysis. Second, for the matched case-control study, the most serious problem was choosing the matching criteria. There is no doubt that the more criteria are chose, the more accurate the result will be. However, if the matching criteria are too stringent, it is difficult to find control patients and further decrease the sample size [22]. Therefore, three core matching variables were chose in our study to maintain the balance between accuracy and maximum sample size.

\section{Conclusions}

The present study assessed the cost attributable to CRBSI and explored its influencing factors. Our findings revealed that CRBSI introduced a heavy economic burden for patients, especially older patients, those with severe conditions (ICU or more catheters), and those infected by fungi. Although the incidence of CRBSI in China has been lower in recent years through measures taken by the government and hospitals, more attentions should be paid to further prevent and control the infection; if possible, the level of medical insurance payment for the CRBSI patients should be increased during the new medical reform, with the purpose of relieving the economic burden among patients in China.

\section{Abbreviations \\ CRBSI: Catheter-related bloodstream infection; CT: Computed tomography; CVC: Central venous catheter; G +: Gram-positive bacterium; G-: Gram- negative bacterium; GLM: General linear model; HIS: Hospital information system; ICU: Intensive care unit}

\section{Acknowledgements}

The authors wish to thank the professors from infection control department in this tertiary hospital for providing important suggestions and their assistants for the support of data collecting.

Funding

This work is funded by Becton, Dickinson and Company. 


\section{Availability of data and materials}

The datasets used during the study are available from the corresponding author on reasonable request.

\section{Authors' contributions}

YC and HW studied the concept and made the whole design.YC, MZ and XC collected the data during the field study.YC and WS conducted the analysis and interpretation of data. $Y C$ were the main drafter of the manuscript. All authors read and approved the final manuscript.

\section{Ethics approval and consent to participate}

Not applicable.

\section{Consent for publication}

All of the data is allowed by the hospital.

\section{Competing interests}

The authors declare that they have no competing interests.

\section{Publisher's Note}

Springer Nature remains neutral with regard to jurisdictional claims in published maps and institutional affiliations.

\section{Author details}

${ }^{1}$ Department of Health Service Management, School of Humanities and Social Sciences, China Medical University, No.77 Puhe Road, Shenyang North New Area, Shenyang, Liaoning Province 110122, People's Republic of China. ${ }^{2}$ Department of Social Medicine, School of Public Health, China Medical University, No.77 Puhe Road, Shenyang North New Area, Shenyang, Liaoning Province 110122, People's Republic of China.

\section{Received: 5 March 2018 Accepted: 26 September 2018}

\section{Published online: 11 October 2018}

\section{References}

1. Goudie A, Dynan L, Brady PW, Rettiganti M. Attributable cost and length of stay for central line-associated bloodstream infections. Pediatrics. 2014; 133(6):1525-32

2. Blot SI, Depuydt P, Annemans L, Benoit D, Hoste E, De Waele JJ, et al. Clinical and economic outcomes in critically III patients with nosocomial catheter-related bloodstream infections. Clin Infect Dis. 2005:41(11):1591-8

3. Nakamura I, Fukushima S, Hayakawa T, Sekiya K, Matsumoto T. The additional costs of catheter-related bloodstream infections in intensive care units. Am J Infect Control. 2015;43(10):1046-9.

4. Warren DK, Quadir WW, Hollenbeak CS, Elward AM, Cox MJ, Fraser VJ. Attributable cost of catheter-associated bloodstream infections among intensive care patients in a nonteaching hospital. Crit Care Med. 2006;34(8): 2084-9.

5. Wilson MZ, Rafferty C, Deeter D, Comito MA, Hollenbeak CS. Attributable costs of central line associated bloodstream infections in a pediatric hematology/oncology population. Am J Infect Control. 2014;42(11):1157-60.

6. Leistner R, Hirsemann E, Bloch A, Gastmeier P, Geffers C. Costs and prolonged length of stay of central venous catheter-associated bloodstream infections (CVC BSI): a matched prospective cohort study. Infection. 2014; 42(1):31-6.

7. Higuera F, Rangel-Frausto MS, Rosenthal VD, Soto JM, Castañon J, Franco G, et al. Attributable cost and length of stay for patients with central venous catheter-associated bloodstream infection in Mexico City intensive care units: a prospective, matched analysis. Infect Control Hosp Epidemiol. 2007; 28(1):31-5.

8. Rosenthal VD, Guzman S, Migone O, Crnich CJ. The attributable cost, length of hospital stay, and mortality of central line-associated bloodstream infection in intensive care departments in Argentina: a prospective, matched analysis. Am J Infect Control. 2003;31(8):475-80

9. Dimick JB, Pelz RK, Consunji R, Swoboda SM, Hendrix CW, Lipsett PA. Increased resource use associated with catheter-related bloodstream infection in the surgical intensive care unit. Arch Surg. 2001;136(2):229-34.

10. Shannon RP, Patel B, Cummins D, Shannon AH, Ganguli G, Lu Y. Economics of central line-associated bloodstream infections. Am J Med Qual. 2006; 21(Suppl 6):7-16.
11. National Health and Family Planning Commission of the People's Republic of China. Guiding opinions on strengthening the performance evaluation of public medical institutions. http://www.nhfpc.gov.cn/renshi/s3577/201512/ d0be65b3c9464923b541890cbfb4204f.shtml. Accessed 5 Oct 2016.

12. Zeng C, Chen YH, Jia HX, Li LY, Wu AH. Investigation of catheter-related bloodstream infections in ICU. Chin J Nosocomiol. 2014;24(21):5304-11 (in Chinese).

13. National Health and Family Planning Commission of the People's Republic of China. Guideline for the Prevention and Control of Catheter-related Bloodstream Infection(for Trial Implementation). http://www.moh.gov.cn/ mohyzs/s3594/201012/50039.shtml. Accessed 12 July 2017.

14. Laupland KB, Lee $H$, Gregson DB, Manns BJ. Cost of intensive care unitacquired bloodstream infections. J Hosp Infect. 2006;63(2):124-32

15. National Health and Family Planning Commission of the People's Republic of China. Regulations of surgical management of medical institutions (for Trial Implementation). http://www.moh.gov.cn/mohyzs/s3586/201208/ 55613.shtml. Accessed 25 July 2017.

16. National Health and Family Planning Commission of the People's Republic of China. Statistical yearbook for health and family planning of China in 2016. Beijing: Peking Union Medical College Press; 2016. p. 114.

17. National Bureau of Statistics of the People's Republic of China. Statistical bulletin of the national economy and social development in 2015. http://www. stats.gov.cn/tjsj/zxfb/201602/t20160229_1323991.html. Accessed 8 Oct 2017.

18. Folland S, Goodman AC, Stano M. The economics of health and health care Beijing: China Renmin University Press; 2011. p. 191-3.

19. Mo CM, Yang TJ. Study on the influencing factors of cost for inpatients in ICU. Chin Heal Quality Manag 2004; 11(4):28-29(in Chinese).

20. Yang B, Xiang YS. The influence factors of ICU catheter related bloodstream infection and prevention and control measures. Chin J Exp Clin Infect Dis (Electronic Edition). 2016;10(4):413-6 (in Chinese).

21. Duan W, Zheng A, Mu X, Li M, Liu C, Huang W, et al. How great is the medical burden of disease on the aged? Research based on "system of health account 2011". Health Qual Life Outcomes. 2017;15(1):134

22. Lauria FN, Angeletti $C$. The impact of nosocomial infections on hospital care costs. Infection. 2003:31(Suppl 2):35-43.
Ready to submit your research? Choose BMC and benefit from:

- fast, convenient online submission

- thorough peer review by experienced researchers in your field

- rapid publication on acceptance

- support for research data, including large and complex data types

- gold Open Access which fosters wider collaboration and increased citations

- maximum visibility for your research: over $100 \mathrm{M}$ website views per year

At BMC, research is always in progress.

Learn more biomedcentral.com/submissions 